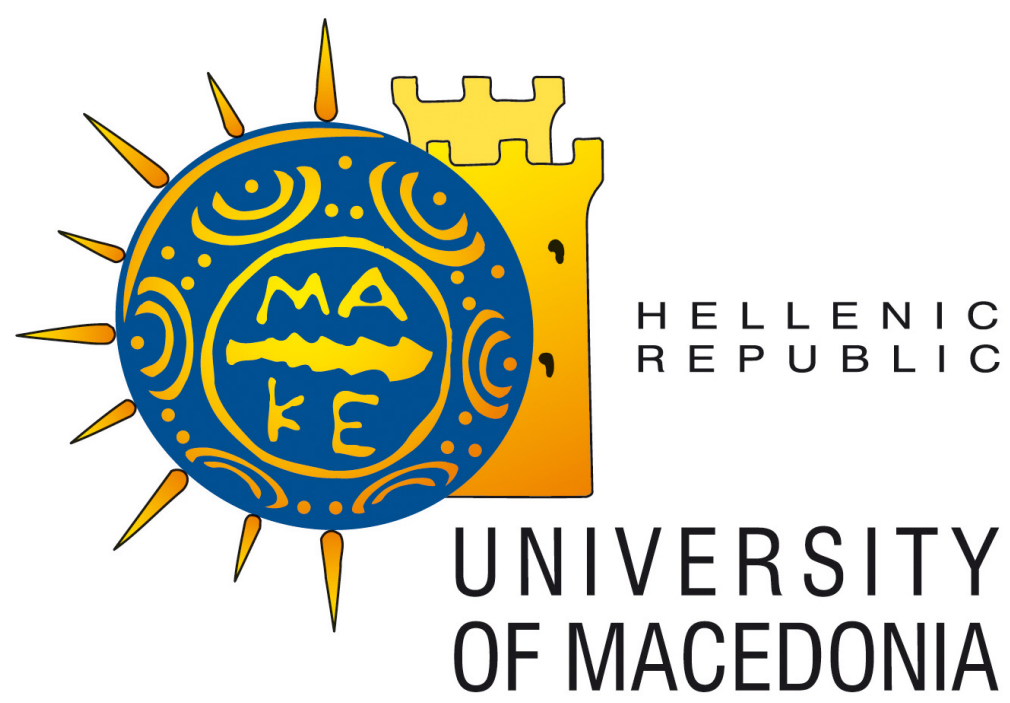

ISSN 1791-3144

\author{
University of Macedonia \\ Department of Economics
}

Discussion Paper Series

\title{
A note on the estimated GARCH coefficients from the S\&P1500 universe
}

Georgios Bampinas, Konstantinos Ladopoulos and Theodore Panagiotidis

\section{Discussion Paper No. 4/2017}




\title{
A note on the estimated GARCH coefficients from the S\&P1500 universe
}

\author{
Georgios Bampinas* \\ Konstantinos Ladopoulos ${ }^{\dagger}$ \\ Theodore Panagiotidis $\ddagger$
}

April 18, 2017

\begin{abstract}
We employ 1440 stocks listed in the S\&P Composite 1500 Index of the NYSE. Three benchmark GARCH models are estimated for the returns of each individual stock under three alternative distributions (Normal, $t$ and GED). We provide summary statistics for all the GARCH coefficients derived from 11520 regressions. The EGARCH model with GED errors emerges as the preferred choice for the individual stocks in the S\&P 1500 universe when non-negativity and stationarity constraints in the conditional variance are imposed. $57 \%$ of the constraint's violations are taking place in the S\&P small cap stocks.
\end{abstract}

Keywords: GARCH, GJR-GARCH, EGARCH, alternative distributions, volatility, time-series

\footnotetext{
*Department of Economics, University of Macedonia, Greece,bampinasg@uom.edu.gr

†Citrix Systems Research \& Development Ltd, Cambridge CB4 0FY, UK, kostaslamda@gmail.com

${ }^{\ddagger}$ Department of Economics, University of Macedonia, Greece, tpanag@uom.edu.gr. Corresponding author, Tel: +302310891736 .
} 


\section{Introduction}

Over the past three decades GARCH models have become a cornerstone of financial econometrics; especially in modeling time series with leptokurtic observations and volatility clusters (Engle, 1982; Bollerslev, 1986). The success of the ARCH/GARCH class of models at capturing these features is well documented (see for example, Bollerslev et al., 1992). However, Bollerslev et al. (1994) argue that a standard GARCH model with normally distributed errors is not sufficient for explaining these two stylized facts of financial time series. Consistent with this notion, Malmsten and Teräsvirta (2004) demonstrate that the latter can be improved by replacing the normal with more fat-tailed distributions. Of importance are also the necessary and sufficient conditions for the stationarity and non-negativity of the conditional variance (see e.g., Lindner, 2008; Teräsvirta. 2008). More recently Ardia and Hoogerheide (2013) examined the cross-sectional distribution of GJR-GARCH-student model parameters over the constituents of the S\&P500 index. They conclude that small cap stocks have higher volatility $!^{1}$

However, little is known about the expected values of the coefficients of alternative GARCH models. For instance, in many cases, initial values are required. Most researchers rely on crude estimates of GARCH parameters, usually summing to less than unity (i.e. $\alpha=0.15, \beta=0.8, \alpha+\beta \prec 1$ ). We fill this gap by providing the average estimated values of the coefficients of the three benchmark GARCH models. We consider the most popular GARCH specifications; the GARCH $(1,1)$ (Bollerslev, 1986), the EGARCH(1,1) (Nelson, 1991) and the GJR-GARCH(1,1) (Glosten et al., 1993). As the risk-return relation depends on the error distribution (Baillie and DeGennaro, 1990), we examine three alternative distributions; the Gaussian normal $(N)$, the Student's- $t(t)$, and the Generalized Error Distribution $(G E D)$. Additionally, examining the stationarity and non-negativity constraints for various GARCH would enlighten our knowledge further on the extend to which the S\&P 1500 stocks satisfy strictly stationarity conditions. We also aim to examine whether these are related to sectoral characteristics and/or to small cap stocks.

This paper focuses on the summary statistics of the estimated coefficients of three benchmark GARCH models. The purpose of our study is threefold: (i) to compare three popular GARCH models in terms of statistical significance of their coefficients (ii) to provide descriptive analysis

\footnotetext{
1 Nelson and Cao (1992) derived the necessary and sufficient conditions for the non-negativity of the conditional variance in higher-order GARCH models. The GARCH(2,2) case has been studied in detail by He and Terasvirta (1999).
} 
for the estimated GARCH coefficients for all common stocks in the S\&P 1500 universe and (iii) examine the extent to which the benchmark GARCH models fulfill the non-negativity and stationarity constraints, under the three alternative distributions. We find that the EGARCH $(1,1)$ model with GED errors is the best model for the daily financial returns of the S\&P 1500 universe (in terms of satisfying both constraints). Once the two constraints are imposed the estimated coefficients show less dispersion. The stocks that did not satisfy the non-negativity and stationarity requirements tend to be drawn from the Technology and the Consumers Services sector.

The rest of the paper is organized as follows: section 2 presents the data, section 3 describes the econometric methodology, section 4 discusses the main results and the last one concludes.

\section{Data}

We use stock prices that have been constituents of the S\&P1500 Composite Index continuously for the period January 2008 until December 2011. For all common stocks, we obtain 1043 daily closing prices (cumulative stock price accounting for dividend gains and splits) from Yahoo Finance (http:/ / finance.yahoo.com/). Our sample consists of 1440 individual stocks; 485 from the S\&P 500 Index, 382 from S\&P MidCap 400 Index and 573 from the S\&P SmallCap 600 Index. Log returns of each individual stock $\left(r_{t}\right)$ are calculated as log differences. Returns are found stationary (i.e. $I(0))$ and leprokurtic ${ }^{2}$

\section{Econometric specification}

We follow the specification of Engle and Patton (2001) and the returns equation is specified as:

$$
r_{t}=c+\varepsilon_{t}
$$

where $\varepsilon_{t}=z_{t} h_{t}^{1 / 2}, r_{t}$ is the daily stock returns, $h_{t}$ is the conditional variance and $c$ the constant term. $\left\{z_{t}\right\}$ is a sequence of independent, iid random variables with zero means and unit variance. This implies that $\varepsilon_{t} \mid \mathcal{F}_{t-1} \stackrel{d}{\sim}\left(0, h_{t}\right)$ where $d$ stand for the distribution (usually assumed normal but we also consider $t$ and GED).

\footnotetext{
${ }^{2}$ The unit root tests and the summary statistics of all series are available from the authors upon request.
} 
For the conditional variance equation three specifications were considered:

$$
\begin{gathered}
\operatorname{GARCH}(1,1): h_{t}=\omega+\alpha \varepsilon_{t-1}^{2}+\beta h_{t-1} \\
\operatorname{GJR}-\operatorname{GARCH}(1,1): h_{t}=\omega+\alpha \varepsilon_{t-1}^{2}+\gamma \varepsilon_{t-1}^{2} I_{t-1}+\beta h_{t-1} \\
\operatorname{EGARCH}(1,1): \log \left(h_{t}\right)=\omega+\alpha\left[\frac{\left|\varepsilon_{t-1}\right|}{\sqrt{h_{t-1}}}-\sqrt{\frac{2}{\pi}}\right]+\gamma \frac{\varepsilon_{t-1}}{\sqrt{h_{t-1}}}+\beta \log \left(h_{t-1}\right)
\end{gathered}
$$

where Eq. (2) is the conditional variance equation of a $\operatorname{GARCH}(1,1)$ model (see Bollerslev, 1986). A sufficient condition for the conditional variance to be positive is $\omega>0, \alpha \geqslant 0, \beta \geqslant 0$. In general, the $\operatorname{GARCH}(1,1)$ process is weakly stationary if and only if $\alpha+\beta<1$. To capture the possibility of asymmetric effects of returns on volatility, we also consider the GJR-GARCH model, introduced by Glosten et al. (1993) and the EGARCH model, proposed by Nelson (1991), shown in equations (3) and (4), respectively. In GJR-GARCH, the indicator function is $I_{t-1}=1$ if $\varepsilon_{t-1}<0$ and 0 otherwise, while $\gamma$ denotes the asymmetry factor (if $\gamma>0$ the negative errors are leveraged). For stationarity, $\alpha+\beta+\gamma / 2<1$ needs to hold (see Engle et al., 2008). Nelson (1991) examined the moments of the general infinite-order Exponential ARCH model $!^{3}$ Provided that all moments of $\left\{\varepsilon_{t}\right\}$ exist, all moments for the $\operatorname{EGARCH}(1,1)$ process exist when $\beta^{2}<1$ is satisfied $(\gamma<0$ for leverage effects). In Eq. (4) no parameter restrictions are necessary to ensure the non-negativity of $h_{t}$.

The three models are estimated using the quasi maximum likelihood estimation method (QMLE) ${ }^{4}$ Nelson (1991) indicates that GED and $N$ are the most preferable cases for GARCH models with the latter to be a special case of the former $(v=2)$. Horvath and Sopov (2016) found that GARCH models with normal distribution underestimate the tail risk and demonstrate that Student's $t$ conditional distributions capture it more accurately. For the EGARCH model, we consider two parametric distributions $(N, G E D)$ while for the GARCH and GJR-GARCH we also consider the Student's $t$. Furthermore, the empirical distribution of the GARCH models parameter estimates will be approximated with a non-parametric kernel fit.

\footnotetext{
${ }^{3}$ The moment structure of the EGARCH(1,1) model has also been discussed in He et al. (2002) and Karanasos and $\operatorname{Kim}(2003)$.

${ }_{4}^{4}$ Bollerslev and Wooldridge (1992), pointed out that the assumption of the normality of the standardized conditional errors may be too strong and can cause misspecification of the likelihood function. To deal with this, they suggest the use of Quasi Maximum Likelihood Estimation (QMLE). All estimations have been carried out in EViews 8.
} 
Concluding, we examine whether each estimated model satisfies the non-negativity and weakly stationarity constraints. We consider the validity of two conditions: (i) compliance to the non-negativity and stationarity requirements and (ii) statistical significance of the coefficients at the $10 \%$ level. If any of the two conditions is not satisfied, the stock is removed from the subsequent analysis.

\section{Empirical results}

We start by running eight (8) alternative GARCH models for each one of the 1440 stocks under examination (totally $1440 \times 8=11520$ regressions). For each stock we get eight estimates of $\omega, \alpha$ and $\beta$ and five estimates for $\gamma$ under the three alternative distributions (normal and GED for the EGARCH specification). The empirical distribution of the GARCH models parameter estimates will be approximated with a non-parametric fit (kernel).

The summary statistics for the coefficients of the models without any restrictions in the conditional variance equation are reported in Table 1 (out of the $1440 \mathrm{GARCH}$ regressions for each model). The average $\omega$ is very close to zero for the GARCH $(1,1)$ and the GJR-GARCH models for all distributions. In the EGARCH model, we observe negative $\omega$ values for both the normal and the GED. Similar results hold for the median $\omega$. Negative skewness is present only in the EGARCH specification while for all models the kurtosis value is high. The mean and median $\alpha^{\prime} s$ is positive and close to zero with the higher observed estimates for the EGARCH model. Higher standard deviation and kurtosis are reported for the GJR-GARCH and EGARCH specifications when the errors are assumed to be normal. In these cases, we also get the higher skewness values (37.6 and 7.67 respectively). The $\beta^{\prime}$ s bear much resemblance amongst all the alternative models and distributions. The mean $\beta$ ranges between $0.85(\mathrm{GARCH}-N)$ and $0.97(\mathrm{EGARCH}-\mathrm{GED})$ while its median value ranges between 0.88 and 0.98 . The $\beta$ standard deviation is higher for the GARCH and EGARCH models with normal errors. For all models, $\beta$ is negatively skewed. The EGARCH model estimates are negatively skewed (-9.77) and leptokurtic (112.04) for the normal and -22.58 (559.79) for the GED. Finally, the $\gamma$ mean value is positive (negative) on average for the GJR-GARCH (EGARCH) under all distributional assumptions. In absolute values, the average $\gamma^{\prime} \mathrm{s}$ range between 0.06 to 0.09 . Higher values of kurtosis and standard deviation along with negative 
skewness are observed for the GJR-GARCH with normal errors.

The next step would be to filter the models and examine only those that comply with the two criteria. The summary statistics for the models that satisfy the non-negativity and stationarity constraints in the conditional variance equation are reported in Table 2. Panel A in Table 2 reports the absolute number and the proportion of estimates that satisfy both conditions for each model. The significance level for each estimated coefficient is set at the $10 \%$. Out of the total of 11520 regressions, almost half (6465) satisfy the non-negativity (for GARCH and GJR-GARCH) and stationarity constraints (56.12\% of all the regressions). The vast majority of GARCH and EGARCH models with GED errors fulfill the two criteria (92.71\% and $84.72 \%$ respectively). Panel B in Table 2 presents the descriptive analysis of all the regressions that satisfy both conditions. In particular, the distribution of the coefficients of GARCH(1,1) and EGARCH following GED appear to be similar to Table 1. One note though is that the skewness of $\gamma$ for GJR-N, EGARCH-N and GED has now the opposite sign. Moreover, the average (absolute) value of $\gamma$ is higher (compared to Table 1) ranging from 0.076 to 0.115 .

Table 3 presents the proportion of statistically significant coefficients at the $1 \%, 5 \%$ and $10 \%$ significance level for the cases that both conditions are satisfied. On the one side of the spectrum is the GJR-GARCH with the lowest proportion of significant coefficients (starting from $9.5 \%$ for $\alpha$, $26.5 \%$ for $\omega, 29.5 \%$ for $\gamma$ ) and EGARCH on the other with the highest (min $62.91 \%$ and the rest considerably higher).

In general, $\operatorname{GARCH}(1,1)$ and $\operatorname{EGARCH}(1,1)$ with GED errors emerge as the two front-runners. For the the former $\omega$ is significant in $23.97 \%$ of the cases, $99.1 \%$ for $\alpha, 99.63 \%$ for $\beta$ in the models that satisfy both conditions. These number for EGARCH are $98 \%, 96 \%, 100 \%$ and $81 \%$ for $\omega, \alpha, \beta$ and $\gamma$ respectively.

Figure 1 presents the histogram of the $\operatorname{EGARCH}(1,1)$ coefficients estimates against the $t$ distribution and a non-parametric kernel fit. The left panel depicts the unrestricted coefficients whereas the right the ones that satisfy the two restrictions. We can observe that the estimated coefficients are concentrated around the mean once the two restrictions are taken into account. As far as $\gamma$ is concerned (the asymmetry coefficient), the second peak of the distribution disappears (see the right panel). In all the cases where the two conditions are met, the distribution appears to be more symmetric. The next step would be to identify whether the stocks that did not satisfy the 
two conditions stem from specific sectors.

Table 4 presents the stocks that did not satisfy the two restrictions under the $\operatorname{EARCH}(1,1)$ GED specification along with their sectoral classification. Out of the 220 stocks that do not satisfy the two conditions, 57\% of them belong to the S\&P SmallCap 600 Index. In other words, lower capitalization firms are more likely to violate the stationarity and statistical significance conditions. Moreover, we observe that most companies (in absolute number) that did not satisfy the two conditions are derived from Technology and Consumer Services sector followed by the Financials and Healthcare sector (these sectors regularly include stocks with higher returns). Finally, intra-sectoral analysis reveals that the highest percentage of stocks that did not satisfy the two restrictions is observed for the Consumer Goods (29.75\%), Consumer Services (20.68\%) and the Healthcare (20.28\%) sector.

\section{Conclusions}

Although the literature on univariate GARCH models is voluminous, we still do not know much about the expected values of their coefficients. We employ 1440 stocks (all belonging to the S\&P 1500) and estimate eight alternative symmetric and asymmetric GARCH models. We examined the summary statistics and statistical significance of the GARCH coefficients $(\omega, \alpha$ and $\beta$ and $\gamma)$ from 11520 regressions. Additionally, we explored whether the estimated models satisfy jointly two conditions: (i) conditional variance stationarity and (ii) coefficients statistical significance at the $10 \%$ level.

We find that EGARCH with GED errors that allow for heavier tails, emerges as the most adequate model to fit the financial returns in the S\&P 1500 universe. This specification produces the highest proportion of significant $\omega, \alpha, \beta$ and $\gamma$, with the Students' $t$ and normal distributions following in order. The GARCH and EGARCH models with GED errors provide the most statistically significant results. For the former, the average values are $\omega=0.00, \alpha=0.094, \beta=0.883$ whereas these numbers for the latter are $\omega=-0.247, \alpha=0.144, \beta=0.981$ and $\gamma=-0.076$ (for those models that satisfy both criteria). These could serve as useful intitial values. Under the GJR-GED specification, only 392 out of the 1440 estimated models are acceptable, while the asymmetry coefficient $\gamma$ was found significant only in $48.47 \%(81.31 \%)$ of the GJR-GED (EGARCH-GED) models. 
Finally, we find that lower capitalization firms are more likely to violate the stationarity and statistical significance conditions. Most of the stocks that did not satisfy the two conditions (57\%) belong to the S\&P 600 Index and are derived from the Technology and the Consumer Services sector.

\section{Acknowledgments}

The authors would like to thank Dimitrios Thomakos for his useful comments and suggestions.

\section{References}

Ardia, D. and Hoogerheide, L.F. (2013) Cross-sectional distribution of GARCH coefficients across S\&P 500 constituents: time variation over the period 2000-2012, Wilmott, 66, 40-45.

Baillie, R. and DeGennaro, R. (1990) Stock returns and volatility, Journal of Financial and Quantitative Analysis, 25, 203-214.

Bollerslev, T. (1986) Generalized autoregressive conditional heteroskedasticity, Journal of Econometrics, 31, 307-327.

Bollerslev, T. (1987) A conditionally heteroscedastic time series model for speculative prices and rates of return, Review of Economics and Statistics, 69, 542-547.

Bollerslev, T., Chou, R.Y. and Kroner, K.F. (1992) ARCH modeling in finance, Journal of Econometrics, 52, 5-59.

Bollerslev, T., Engle, R.F. and Nelson, D.B. (1994) ARCH Models. In: Engle, R.F. and McFadden, D.L. (Eds.): Handbook of Econometrics, volume 4, 2959-3038. North- Holland, Amsterdam.

Bollerslev, T. and Wooldridge, J.M. (1992) Quasi-maximum likelihood estimation and inference in dynamic models with time-varying covariances, Econometric Reviews, 11, 43-72.

Engle, R.F. (1982) Autoregressive Conditional Heteroscedasticity with Estimates of the Variance of United Kingdom Inflation, Econometrica, 50, 987-1007. 
Engle, R.F., Focardi, M.S. and Fabozzi, J.F. (2008) ARCH/GARCH Models in Applied Financial Econometrics, in Chapter in Handbook Series in Finance by Frank J. Fabozzi (John Wiley \& Sons).

Engle, R.F and Patton, A.J. (2001) What good is a volatility model? Quantitative Finance, 1, 237-245.

Glosten, L.R., Jagannathan, R. and Runkle, D.E. (1993) On the relation between the expected value and the volatility of the nominal excess return on stocks, Journal of Finance, 48, 1779-1801.

He, C. and Terasvirta, T. (1999) Properties of the Autocorrelation Function of Squared Observations for Second Order GARCH Processes under Two Sets of Parameter Constraints, Journal of Time Series Analysis, 20, 23-30.

He, C., Teräsvirta, T. and Malmsten, H. (2002) Moment structure of a family of first-order exponential GARCH models, Econometric Theory, 18, 868-85.

Horvath, R. and Sopov, B. (2016) GARCH models, tail indexes and error distributions: An empirical investigation, North American Journal of Economics and Finance, 37, 1-15.

Karanasos, M. and Kim, J. (2003) Moments of the ARIMA-EGARCH model, Econometrics Journal, $6,146-66$.

Lindner, A.M. (2008) Stationarity, mixing, distributional properties and moments of GARCH(p,q)-processes. In: Andersen, T. G., Davis, R. A., Kreiss, J.-P. and Mikosch, T. (Eds.): Handbook of Financial Time Series, 43-69. Springer, New York.

Maekawa, K., Lee, S. and Tokutsu, Y. (2014) A Note on Volatility Persistence and Structural Changes in GARCH Models, Working Paper.

Malmsten, H. and Teräsvirta, T. (2004) Stylized Facts of Financial Time Series and Three Popular Models of Volatility. SSE/EFI Working Paper Series in Economics and Finance, Stockholm School of Economics 563.

Nelson, D.B., (1991) Conditional Heteroskedasticity in Asset Returns: A New Approach, Econometrica, 59, 347-370. 
Nelson, D.B. and Cao, C.Q. (1992) Inequality Constraints in the Univariate GARCH Model, Journal of Business and Economic Statistics, 10, 229-235.

Teräsvirta, T. (2008) An introduction to univariate GARCH models. In: Andersen, T.G., Davis, R.A., Kreiss, J.-P. and Mikosch, T. (Eds.): Handbook of Financial Time Series, 17-42. Springer, New York. 
Table 1: Summary statistics for the estimates of alternative GARCH models in the S\&P 1500 universe. The table reports descriptive statistics of the estimated coefficients of the GARCH models (1440 for each of the three cases) (see Eq. (2), Eq (3), Eq(4)) over the sample period Jan. 2008 to Dec. 2011 (daily observations).

\begin{tabular}{|c|c|c|c|c|c|c|c|c|}
\hline & \multicolumn{3}{|c|}{ GARCH (1.1) } & \multicolumn{3}{|c|}{ (GJR-GARCH(1,1) } & \multicolumn{2}{|c|}{ EGARCH (1.1) } \\
\hline & Normal & Student's $t$ & GED & Normal & Student's $t$ & GED & Normal & GED \\
\hline \multicolumn{9}{|c|}{ Mean } \\
\hline$\omega$ & 0.000 & 0.000 & 0.000 & 0.000 & 0.000 & 0.000 & -0.403 & -0.278 \\
\hline$\alpha$ & 0.110 & 0.090 & 0.094 & 0.083 & 0.036 & 0.036 & 0.157 & 0.145 \\
\hline$\beta$ & 0.855 & 0.894 & 0.884 & 0.878 & 0.905 & 0.896 & 0.959 & 0.976 \\
\hline$\gamma$ & & & & 0.061 & 0.088 & 0.089 & -0.070 & -0.069 \\
\hline \multicolumn{9}{|c|}{ Median } \\
\hline$\omega$ & 0.000 & 0.000 & 0.000 & 0.000 & 0.000 & 0.000 & -0.257 & -0.230 \\
\hline$\alpha$ & 0.093 & 0.082 & 0.085 & 0.028 & 0.028 & 0.027 & 0.145 & 0.142 \\
\hline$\beta$ & 0.887 & 0.907 & 0.901 & 0.905 & 0.917 & 0.913 & 0.980 & 0.983 \\
\hline$\gamma$ & & & & 0.092 & 0.087 & 0.087 & -0.073 & -0.070 \\
\hline \multicolumn{9}{|c|}{ Standard Deviation } \\
\hline$\omega$ & 0.000 & 0.000 & 0.000 & 0.000 & 0.000 & 0.000 & 0.913 & 0.503 \\
\hline$\alpha$ & 0.089 & 0.046 & 0.048 & 1.438 & 0.041 & 0.046 & 0.112 & 0.059 \\
\hline$\beta$ & 0.154 & 0.072 & 0.088 & 0.115 & 0.064 & 0.081 & 0.126 & 0.073 \\
\hline$\gamma$ & & & & 1.437 & 0.052 & 0.056 & 0.062 & 0.030 \\
\hline \multicolumn{9}{|c|}{ Skewness } \\
\hline$\omega$ & 9.277 & 11.824 & 11.693 & 8.248 & 10.467 & 29.189 & -9.801 & -20.71 \\
\hline$\alpha$ & 5.121 & 2.541 & 2.261 & 37.605 & 2.663 & 3.181 & 7.672 & 0.588 \\
\hline$\beta$ & -6.296 & -5.670 & -5.235 & -4.036 & -5.367 & -4.922 & -9.779 & -22.582 \\
\hline$\gamma$ & & & & -37.413 & 0.450 & 0.641 & 9.061 & 0.216 \\
\hline \multicolumn{9}{|c|}{ Kurtosis } \\
\hline$\omega$ & 109.470 & 195.498 & 182.949 & 93.841 & 157.01 & 975.02 & 116.041 & 487.02 \\
\hline$\alpha$ & 49.090 & 14.831 & 12.573 & 1422.50 & 15.442 & 21.334 & 140.074 & 4.694 \\
\hline$\beta$ & 59.041 & 56.367 & 45.336 & 24.807 & 56.669 & 42.585 & 112.041 & 559.79 \\
\hline$\gamma$ & & & & 1413.15 & 6.951 & 8.042 & 196.825 & 3.644 \\
\hline
\end{tabular}


Table 2: Summary statistics for the models that satisfy the conditions: (i) conditional variance stationarity and (ii) coefficients significant at the $10 \%$. The table reports descriptive statistics of the estimated coefficients of the GARCH models (see Eq. (2), Eq (3), Eq(4)) that are significant at $10 \%$ level and also satisfy the condition of variance stationarity in each case. The sample period spans from Jan. 2008 to Dec. 2011.

\begin{tabular}{|c|c|c|c|c|c|c|c|c|}
\hline & \multicolumn{3}{|c|}{ GARCH (1.1) } & \multicolumn{3}{|c|}{ GJR-GARCH(1,1) } & \multicolumn{2}{|c|}{ 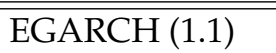 } \\
\hline & Normal & Student's $t$ & GED & Normal & Student's $t$ & GED & Normal & GED \\
\hline \multicolumn{9}{|c|}{ Panel A: Models estimates that satisfy both condition } \\
\hline Count(\#) & $\begin{array}{l}861 \\
5979 \%\end{array}$ & $\begin{array}{l}1147 \\
7965 \%\end{array}$ & $\begin{array}{l}1335 \\
9271^{\circ}\end{array}$ & $\begin{array}{l}200 \\
1389 \%\end{array}$ & $\begin{array}{l}388 \\
2694 \%\end{array}$ & 392 & 922 & $\begin{array}{l}1220 \\
8472 \%\end{array}$ \\
\hline
\end{tabular}

Panel B: Descriptive statistics for the coefficients of the models that satisfy both conditions Mean

\begin{tabular}{lllllllll}
$\omega$ & 0.000 & 0.000 & 0.000 & 0.000 & 0.000 & 0.000 & -0.297 & -0.247 \\
$\alpha$ & 0.114 & 0.093 & 0.094 & 0.054 & 0.053 & 0.055 & 0.151 & 0.144 \\
$\beta$ & 0.857 & 0.889 & 0.883 & 0.868 & 0.884 & 0.879 & 0.975 & 0.981 \\
$\gamma$ & & & & 0.115 & 0.093 & 0.095 & -0.089 & -0.076 \\
Median & & & & & & & & \\
$\omega$ & 0.000 & 0.000 & 0.000 & 0.000 & 0.000 & 0.000 & -0.252 & -0.228 \\
$\alpha$ & 0.104 & 0.086 & 0.086 & 0.048 & 0.047 & 0.047 & 0.144 & 0.142 \\
$\beta$ & 0.878 & 0.903 & 0.900 & 0.880 & 0.896 & 0.893 & 0.982 & 0.984 \\
$\gamma$ & & & & 0.105 & 0.091 & 0.091 & -0.085 & -0.074 \\
Standard Deviation & & & & & & & \\
$\omega$ & 0.000 & 0.000 & 0.000 & 0.000 & 0.000 & 0.000 & 0.265 & 0.119 \\
$\alpha$ & 0.056 & 0.041 & 0.046 & 0.023 & 0.023 & 0.029 & 0.075 & 0.059 \\
$\beta$ & 0.079 & 0.061 & 0.075 & 0.063 & 0.051 & 0.064 & 0.030 & 0.012 \\
$\gamma$ & & & & 0.068 & 0.045 & 0.054 & 0.037 & 0.024 \\
Skewness & & & & & & & & \\
$\omega$ & 14.777 & 7.191 & 12.358 & 5.426 & 5.925 & 5.762 & -8.429 & -2.746 \\
$\alpha$ & 2.802 & 2.439 & 2.311 & 2.204 & 1.891 & 3.287 & 3.701 & 0.394 \\
$\beta$ & -2.670 & -3.823 & -3.792 & -4.046 & -2.384 & -3.462 & -9.709 & -3.832 \\
$\gamma$ & & & & 4.083 & 0.137 & 0.687 & -0.149 & -0.506 \\
Kurtosis & & & & & & & & \\
$\omega$ & 317.602 & 73.215 & 229.798 & 41.604 & 47.827 & 46.996 & 111.122 & 19.464 \\
$\alpha$ & 15.670 & 13.656 & 13.250 & 10.781 & 8.729 & 22.016 & 35.310 & 3.226 \\
$\beta$ & 12.758 & 25.495 & 23.527 & 30.224 & 11.785 & 21.285 & 138.563 & 28.887 \\
$\gamma$ & & & & 36.837 & 7.922 & 12.612 & 50.521 & 3.275 \\
\hline \hline
\end{tabular}


Table 3: Proportion of statistical significant coefficients in the models that satisfy both conditions (i) conditional variance stationarity and (ii) coefficients significant at the $10 \%$. The table reports the proportion (in percent) of the estimated coefficients of the three GARCH models that satisfy both conditions.

\begin{tabular}{|c|c|c|c|c|c|c|c|c|c|c|c|c|}
\hline & \multicolumn{4}{|l|}{ Normal } & \multicolumn{4}{|c|}{ Student's $t$} & \multicolumn{4}{|l|}{ GED } \\
\hline & $\omega$ & $\alpha$ & $\beta$ & $\gamma$ & $\omega$ & $\alpha$ & $\beta$ & $\gamma$ & $\omega$ & $\alpha$ & $\beta$ & $\gamma$ \\
\hline \multicolumn{13}{|c|}{ Panel A: GARCH $(1,1)$} \\
\hline $1 \%$ & $12.43 \%$ & $90.71 \%$ & $100 \%$ & & $18.4 \%$ & $99.65 \%$ & $100 \%$ & & $23.97 \%$ & $99.18 \%$ & $99.63 \%$ & \\
\hline $5 \%$ & $61.79 \%$ & $98.72 \%$ & $100 \%$ & & $69.75 \%$ & $99.91 \%$ & $100 \%$ & & $79.1 \%$ & $99.93 \%$ & $99.93 \%$ & \\
\hline $10 \%$ & $100 \%$ & $100 \%$ & $100 \%$ & & $100 \%$ & $100 \%$ & $100 \%$ & & $100 \%$ & $100 \%$ & $100 \%$ & \\
\hline \multicolumn{13}{|c|}{ Panel B: GJR-GARCH $(1,1)$} \\
\hline $1 \%$ & $26.5 \%$ & $9.5 \%$ & $99.5 \%$ & $29.5 \%$ & $37.11 \%$ & $17.27 \%$ & $100 \%$ & $43.3 \%$ & $43.37 \%$ & $23.21 \%$ & $100 \%$ & $48.47 \%$ \\
\hline $5 \%$ & $75 \%$ & $55 \%$ & $100 \%$ & $72 \%$ & $85.31 \%$ & $64.43 \%$ & $100 \%$ & $83.25 \%$ & $90.31 \%$ & $64.80 \%$ & $100 \%$ & $83.42 \%$ \\
\hline $10 \%$ & $100 \%$ & $100 \%$ & $100 \%$ & & $100 \%$ & $100 \%$ & $100 \%$ & $100 \%$ & $100 \%$ & $100 \%$ & $100 \%$ & $100 \%$ \\
\hline \multicolumn{13}{|c|}{ Panel C: EGARCH(1,1) } \\
\hline $1 \%$ & $78.53 \%$ & $84.27 \%$ & $100 \%$ & $62.91 \%$ & & & & & $98.36 \%$ & $96.72 \%$ & $100 \%$ & $81.31 \%$ \\
\hline $5 \%$ & $96.53 \%$ & $96.1 \%$ & $100 \%$ & $88.83 \%$ & & & & & $99.92 \%$ & $99.43 \%$ & $100 \%$ & $93.85 \%$ \\
\hline $10 \%$ & $100 \%$ & $100 \%$ & $100 \%$ & $100 \%$ & & & & & $100 \%$ & $100 \%$ & $100 \%$ & $100 \%$ \\
\hline
\end{tabular}


Table 4: This table presents the stocks that did not satisfy the two restrictions for the EGARCH-GED model. Sectoral information and stock tickers are also provided. Count $(\%)$ denotes the percentage of stocks that did not satisfy the two restrictions in each sector.

\begin{tabular}{|c|c|c|}
\hline Sectors & Tickers & Count $(\%)$ \\
\hline Basic Materials & AVD, CENX, WKN, RDK, SWM, VMC, WOR, ZEP & $8.8 \%$ \\
\hline Cons. Goods & $\begin{array}{l}\text { AVP, BGS, CALM, DF, DMND, FLO, GMCR, HRL, HSY, KR, LNCE, MKC, NAFC,PBH, PEET, } \\
\text { RAH, SAM, SENEA, SPTN, SVU, SWY, WDFC }\end{array}$ & $29.75 \%$ \\
\hline Cons. Services & $\begin{array}{l}\text { AAN, AEO, APOL, BBY, BIG, BKE, BTH, CBK, CECO, CPLA, CRI, CROX, CSTR, CWTR, DHI } \\
\text { DV, DW, EXBD, F, HHS, HOTT, HVT, ISCA, JOSB, KIRK, LQDT, LTM, MCRI, MDC, NFLX, NILE, } \\
\text { NTRI, NYT, PBY, PETS, RGR, RSH, SHFL, SHLD, SONC, SPF, STMP, STRA, THO, TWC, WGO, } \\
\text { URBN, WHR, ZUMZ }\end{array}$ & $20.68 \%$ \\
\hline Energy & CKH, HFC, TDW, TSO, VLO, WFR & $6.82 \%$ \\
\hline Financials & $\begin{array}{l}\text { AIG, AIZ, AJG, AMSF, AVB, BKMU, BPFH, BXS, CATY, ESS, EZPW, FMER, FNF,.GBCI, GHL, } \\
\text { GTY, HAFC, HBHC, HOMB, IBKR, NAVG, NYB, ONB, ORI, ORIT, PFS, PLFE, STBA, SUSQ, } \\
\text { TCB, TWGP, UCBI, UFCS, WTFC }\end{array}$ & $13.23 \%$ \\
\hline Healthcare & $\begin{array}{l}\text { AFAM, AFFX, BIIB, BSX, CBST, CMN, CPSI, CVH, CYH, GB, HAE, HITK, HRC, HWAY, ICUI } \\
\text { KNSY, LH, MNTA, NUVA, OMI, PMTI, QCOR, SLXP, SRDX, SVNT, THC, THOR, VIVO, VPHM }\end{array}$ & $20.28 \%$ \\
\hline Industrials & $\begin{array}{l}\text { CASY, CDI, CLC, CPRT, DNB, FCN, HSII, HTLD, IRM, ITT, KNX, LAWS, LDL, NCI, OSK, PBI, } \\
\text { SSD, TBI, TNC, WERN, WIRE }\end{array}$ & $10.29 \%$ \\
\hline Technology & $\begin{array}{l}\text { ACXM, ADI, ADTN, AKAM, AMD, APH, AVID, CDNS, CMTL, CRM, CSC, CSCO, CSGS ,DGII, } \\
\text { DSPG, DTSI, EBIX, EBS, EQIX, FISV, GOOG, ININ, IVAC, LXK, MCHP, MCRL, MSTR, NANO, } \\
\text { NCIT, NSIT, NTLS, NVDA, NVTL, QLGC, RSTI, SSYS, SUPX, SYNA, TLAB, TQNT, TSRA,TYL } \\
\text { TTWO, VSAT, ZBRA }\end{array}$ & $18.29 \%$ \\
\hline Telecommunications & INSP, PCS, USMO, VCI & $20.00 \%$ \\
\hline Utilities & CHG, PCG & $3.08 \%$ \\
\hline
\end{tabular}




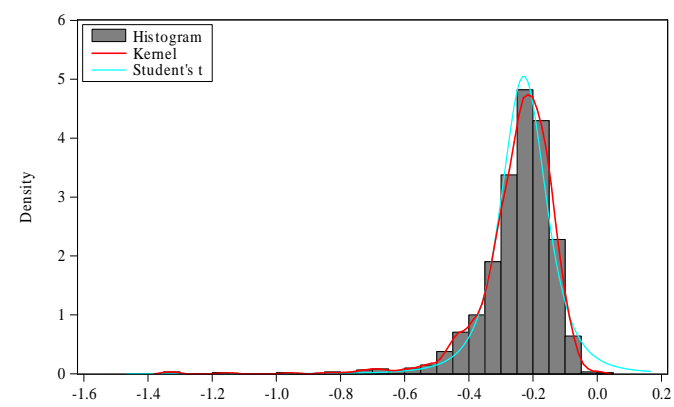

(a) omega

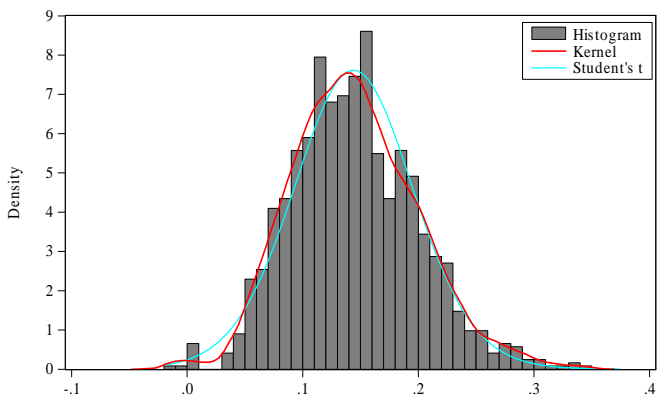

(c) alpha

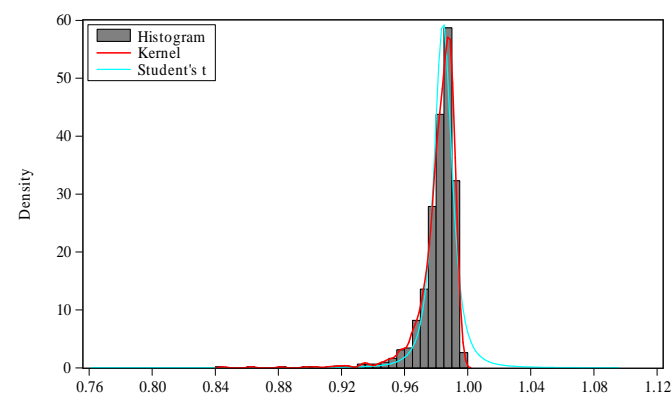

(e) beta

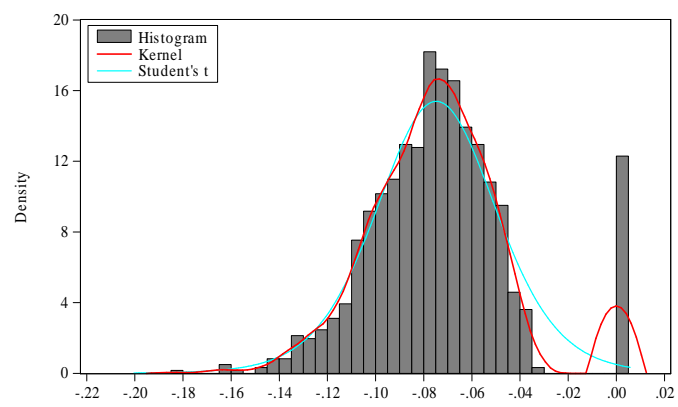

(g) gamma

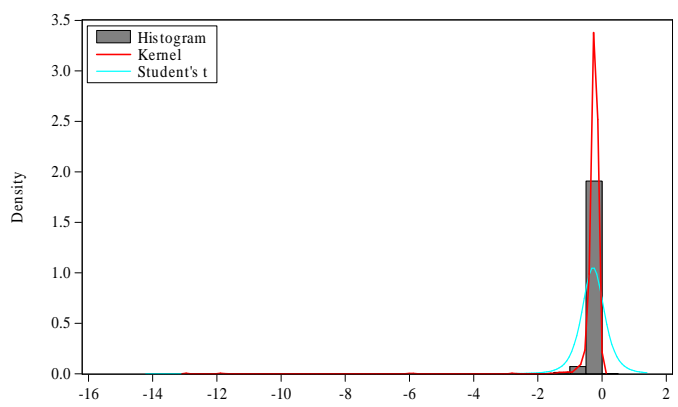

(b) omega viable

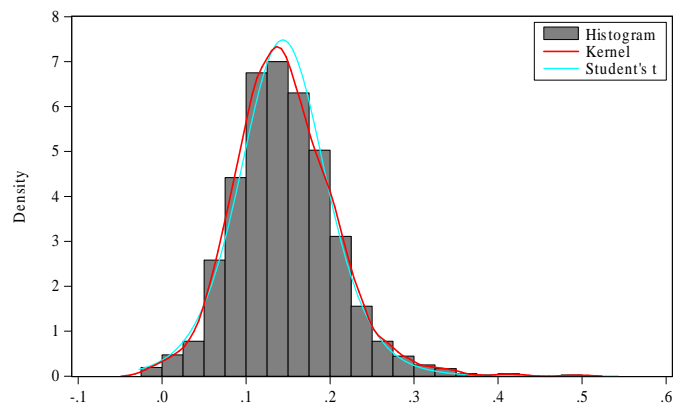

(d) alpha viable

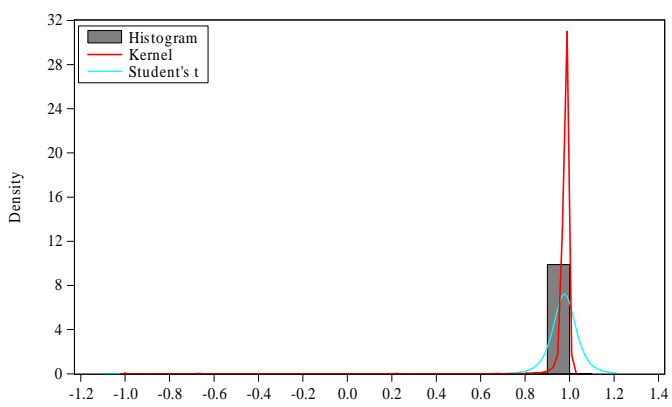

(f) beta viable

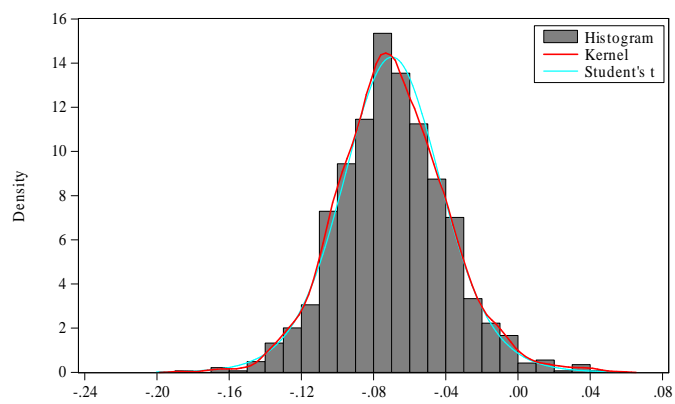

(h) gamma viable

Figure 1: EGARCH(1,1)-GED coefficients distribution with (right panel) and without (left panel) restrictions in conditional variance. 